\title{
Nanotechnology: the scope and potential applications in orthopedic surgery
}

\author{
Ashok Gavaskar ${ }^{1}$ D. Rojas ${ }^{2}$ - F. Videla ${ }^{3}$
}

Received: 20 March 2018 / Accepted: 21 March 2018 / Published online: 30 March 2018

○) Springer-Verlag France SAS, part of Springer Nature 2018

\begin{abstract}
Nanotechnology involves manipulation of matter measuring 1-100 $\mathrm{nm}$ in at least one of its dimensions at the molecular level. Engineering and manipulation of matter at the molecular level has several advantages in the field of medicine (nanomedicine) since most of the biological molecules exist and function at a nanoscale. Though promising, questions still remain on how much of this will ultimately translate into achieving better patient care. Concerns of cost-effectiveness and nanotechnology safety still remain unclear. Orthopedics is an attractive area for the application of nanotechnology since the bone, and its constituents such as hydroxyapatite, Haversian systems, and the collagen fibrils are nanocompounds. The major orthopedic applications of nanotechnology involve around (i) effective drug delivery systems for antibiotics and chemotherapeutic agents, (ii) surface preparation of implants and prosthesis to improve osteointegration and reduce biofilm formation, (iii) controlled drug eluting systems to combat implant-related infections, (iv) tissue engineering for scaffolds preparation to deal with bone and cartilage defects, and (v) diagnostic applications in the field of oncology and musculoskeletal infections.
\end{abstract}

Keywords Nanotechnology $\cdot$ Nanomedicine $\cdot$ Orthopedics $\cdot$ Tissue engineering

\section{Introduction}

The potential positive implications of nanoconcepts were appreciated as early as 1959 by Richard Feynman. The word nanotechnology was coined much later by Norio Taniguchi in 1974, and the concept became popular with the works of Eric Drexler [1]. Nanotechnology involves manipulation of matter measuring $1-100 \mathrm{~nm}$ in at least one of its dimensions at the molecular level. Engineering and manipulation of matter at the molecular level has several advantages in the field of medicine (nanomedicine) since most of the biological molecules exist and function at a nanoscale. Though promising, questions still remain on how much of this will ultimately translate into achieving better patient care. Concerns

Ashok Gavaskar

gavaskar.ortho@gmail.com

1 Department of Orthopaedics, Gleneagles Global Health City, Chennai 600105, India

2 Department of Orthopaedics, Denver Health Medical Center, 777 Bannock Street, Denver, CO 80204, USA

3 Department of Orthopaedics, Fundación Sanatorio Güemes, F. Acuña de Figueroa 1240, Buenos Aires, Argentina of cost-effectiveness and nanotechnology safety still remain unclear.

Orthopedics is an attractive area for the application of nanotechnology since the bone and its constituents such as hydroxyapatite, Haversian systems, and the collagen fibrils are nanocompounds. In orthopedic surgery, there is often a complex interaction between biomaterials and host tissue happening at a micro-level. The effectiveness of such interactions can be significantly improved with material modifications at a nanolevel, using biomaterials created from nanoparticles and structures. This forms the basis for most of the nanotechnology applications in orthopedic science. The application of nanotechnology in orthopedic research is promising because it opens up the opportunity to improve the mechanical properties and biocompatibility of orthopedic-related implantable devices. Nanostructured implants and prosthesis provide with superior mechanical strength, greater wear and corrosion resistance, and potential for drug delivery, and perhaps can act as scaffolds for tissue regeneration [2-4].

The major orthopedic applications of nanotechnology involve around (i) effective drug delivery systems for antibiotics and chemotherapeutic agents, (ii) surface preparation of implants and prosthesis to improve osteointegration 
and reduce biofilm formation, (iii) controlled drug eluting systems to combat implant-related infections, (iv) tissue engineering for scaffolds preparation to deal with bone and cartilage defects, and (v) diagnostic applications in the field of oncology and musculoskeletal infections [5].

\section{Nanotechnology in prosthetic joint replacement}

Failure of osteointegration is one of the main areas of concern with the increasing use of uncemented total joint arthroplasties. Even though the prosthetic joints are currently treated for surface roughness to improve osseous ingrowth or ongrowth, the surface is still smooth at the nanolevel where the cellular interactions take place. This encourages fibrous rather than bony ingrowth leading to premature failure [6]. The use of nanotextured surfaces and nanoengineered implants will help overcome the problem by improving the osteoblastic cellular activity. The enhanced surface area resulting from the reduced grain size of nanoengineering on implants allows superior interactions between the host bone and the implant surface thereby paving the way for a stable and predictable osteointegration, therefore improving implants lifespan.

Periprosthetic joint infection has been one of the leading causes for early failure and revision joint replacement. Various techniques such as antibiotic-loaded cement and other local drug delivery systems have been used with variable rates of success. Use of titanium nanotubes to coat prosthetic surfaces with nanophase silver, or polypeptide nanofilm coatings on prosthetic joint surfaces for controlled and sustained release of antibiotics post-surgery has been described to be successful. Reduction in bacterial adhesion as well as colonization has been documented. Consequently, controlled antibiotic eluting nanophasic prosthetic joints is one potential solution to overcome the catastrophic menace of periprosthetic joint infections [7].

\section{Nanotechnology as a drug delivery system}

Though, treatment and prophylaxis against implant-related and prosthetic joint infection have been the prime focus of nanophase drug delivery systems. This innovative technique have also been found to be beneficial in diagnostic imaging modalities and cancer treatment through a more directed attack on cancer cell, and can also improve the bone formation if used with anabolic agents to minimize osteolysis around the prosthetic joint surfaces [8]. Another promising area for research is the development of injectable drugs loaded in nanospheres that are able to prolong the pharmacological effects of a drug. This can significantly improve the efficacy of intra- and extra-articular (biological/non-biological) injections used for synovitis, arthritic conditions, and tendinopathies.

\section{Nanotechnology and orthopedic oncology}

The orthopedic oncological applications of nanotechnology has major potentials to improve diagnosis, overcome drug resistance, minimize systemic toxicity to normal host cells, and more effectively deliver drugs to cancer cells [9]. Nanoparticles have the ability to carry ligands. Adding specific ligands that bind to the unique genes expressed by tumor cells could improve the ability for an early and precise diagnosis of primary and metastatic malignant bone tumors. Loading contrast agents to the nanoparticles can help with targeted tumor imaging accuracy and to assess tumor viability, which may be very useful for preoperative assessment and surgical mapping. Cancer cells develop resistance by expressing multidrug resistance proteins (MDR) on their surface which pumps the cancer drug out of the cells, thereby reducing its intracellular concentration. Nanotechnology can produce vehicles to effectively deliver cancer drugs into the cell while also carrying specific gene sequences to overcome the MDR proteins. Nanophasic drug delivery systems improve both active and passive targeting of tumor cells. A drug-loaded nanoparticle can be coupled with surface ligands like mannose and folic acid (active targeting) to identify the target cancer cell following endocytosis. Nanoparticles also allow higher drug concentrations inside cancer cells by virtue of their smaller size (passive targeting) and by taking advantage of cancer cells permeability. Nanotechnology can also improve our ability to inhibit the process cancer initiation by downregulating certain genes. Specific molecular markers and fusion oncogenes associated with osteosarcoma and Ewing's sarcoma, respectively, can be downregulated by using nanostructures loaded with particles targeted to inhibit these markers.

\section{Nanotechnology in osseous and chondral defects}

Treatment of segmental bone defects following trauma, failed fixations, and arthroplasty represent a huge challenge. Current modes of addressing these defects using auto/allografts and substitution with porous metals have their own limitations such as limited availability, risk of infection, and poor scaffolding properties, limiting the extent of osteointegration. Since the ideal scaffold used to promote osteointegration will depend on the extent of interactions between the host tissues and the biomaterial, nanostructured biomaterials are ideal to use as these scaffold can be inhabited 
by osteoblasts [10]. The ultimate scaffolds should be biodegradable and act essentially as an extra-cellular matrix on which cells can interact, replicate, and transform into natural tissues.

Nanostructured biomaterials have the ability to provide structural support and optimal pore size and act as a substrate for cell migration and activity. They can also provide biochemical signals when loaded with chemokines and growth factors to control tissue transformation, and finally biological support by presenting peptide sequences to bind receptors and activate intracellular signaling pathways. These properties of nanomaterials are considered ideal when it comes to dealing with large bone defects [11]. Nanoscaffolds will resorb over time once its structural, biological, biochemical, and templating functions have finalized, allowing for a more natural reconstruction without the problems associated with grafts and non-disintegrable biomaterials [12].

Several naturally occurring and synthetic nanostructured materials have been tested for the treatment of bone defects. Natural biomaterials have the advantage of excellent biocompatibility but suffer from poor handling characteristics and reduced structural support. Synthetic materials on the other hand provide excellent structural support but have poor biocompatibility. Currently synthetic biomaterials like bioactive ceramics (tricalcium phosphate (TCP) and hydroxyapatite (HA) and derivatives), polymers like polylactic acid (PLA) and poly-glycolic acid (PGA), and a combination of these called composite matrices are preferred as scaffolding materials for treating bony defects on virtue of their ability to provide better structural support. Surface treatment of these nanostructured biomaterials with growth factors such as bone morphogenic proteins (BMP) and bone sialoproteins (BSP) can improve the ability to achieve successful osteointegration. Natural polymers like fibrin and gelatin have also been used for bone defects in non-weightbearing regions like skull defects.

Cartilage has a more complex structure, and the treatment of cartilaginous defects by using synthetic or biological scaffolds is a bigger challenge. Biological protein scaffolds like collagen and polysaccharide scaffolds like hyaluronic acid, chitosan, chondroitin sulfate, and agarose are preferred for treating cartilage defects because of their excellent biocompatibility, biodegradability, cell infiltration, and neovascularization [13]. Type I collagen scaffolds are the most popular despite their immunoreactivity. Acid-treated collagen gels loaded with mesenchymal stem cells have been shown to produce hyaline-like cartilage in patients with chondral defects. Gelatin is a denatured alternate to collagen without problems of immunoreactivity and disease transmission.

Since most of the cartilage defects are amenable for mini-invasive surgical techniques, availability of scaffolds in injectable form assumes importance. Hydrogels are prepared as nanosized polymeric networks from collagen or gelatin in injectable form with an ability to solidify after implantation and assume the desired shape of the defect. When armed with chondrocytes and injected, hydrogels have been shown to produce cartilage like extra-cellular matrix with progressive improvement in mechanical properties due to continuous deposition of glycosaminoglycanrich matrix.

Uses of nanofibers to produce osteogenic or chondrogenic scaffolds have shown several advantages such as improved cell adhesion, proliferation, and migration. Nanofiber scaffolds have exhibited the highest concentration of collagen type II, improved ability to adsorb human serum proteins, and significant upregulation of cartilagespecific genes and proteins such as collagen II and IX. Several published results have shown that tissue engineering to address cartilage and osseous defects is one of the most important applications for nanotechnology and related research in orthopedics [14].

\section{Future directions}

Nanotechnology has achieved a firm foothold in medicine, and a lot of funding is being allocated for research into nanomedicine. Though the theoretical benefits of nanotechnology has been outstanding, most of the successful in vitro and laboratory-based research have not yet translated into clinical practice. There are concerns about the toxic nature of nanoparticles that are generated as wear debris. Metals tend to behave differently and exhibit different material properties at the nanoscale compared to micro-level. The metal ion particles that have created havoc with metal-on-metal (MOM) hip replacements are in the nanoscale. So current conventional implants treated with nanotechnology for certain specific features are preferred rather than nanoparticles assembled as implants. This avoids the problem of nanoparticles becoming loose and causing tissue toxicity. In view of these issues, a need for regulation has been proposed. Companies are still reluctant to manufacture nanostructured implants and prosthesis given the still unproven clinical advantages, potential risk of toxicity, and the huge costs involved. To conclude, we believe nanotechnology advancements will continue to impact the future of medicine and in particular the orthopedic field. To realize the potential clinical advantages, further research is necessary which needs controlled regulation without stifling the avenues for research.

\section{Compliance with ethical standards}

Conflict of interest The authors indicate no potential conflicts of interest. 


\section{References}

1. Sullivan MP, McHale KJ, Parvizi J, Mehta S (2014) Nanotechnology: current concepts in orthopaedic surgery and future directions. Bone Joint J 96(5):569-573

2. Kienapfel H, Sprey C, Wilke A, Griss P (1999) Implant fixation by bone ingrowth. J Arthroplasty 14(3):355-368

3. Bishop JA, Palanca AA, Bellino MJ, Lowenberg DW (2012) Assessment of compromised fracture healing. J Am Acad Orthop Surg 20(5):273-282

4. Hanc M, Fokter SK, Vogrin M et al (2016) Porous tantalum in spinal surgery: an overview. Eur J Orthop Surg Traumatol 26(1):1-7

5. Garimella R, Eltorai AE (2017) Nanotechnology in orthopedics. J Orthop 14(1):30-33

6. Katz D, Kany J, Valenti P et al (2013) New design of a cementless glenoid component in unconstrained shoulder arthroplasty: a prospective medium term analysis of 143 cases. Eur J Orthop Surg Traumatol 23(1):27-34

7. Gusic N, Ivkovic A, VaFaye J et al (2014) Nanotechnology and bone regeneration: a mini review. Int Orthop 38(9):1877-1884

8. Puckett SD, Taylor E, Raimondo T, Webster TJ (2010) The relationship between the nanostructure of titanium surfaces and bacterial attachment. Biomaterials 31(4):706-713
9. Savvidou OD, Bolia IK, Chloros GD et al (2016) Applied nanotechnology and nanoscience in orthopaedic oncology. Orthopedics 39(5):280-286

10. Andreacchio A, Alberghina F, Testa G, Canavese F (2018) Surgical treatment for symptomatic non-ossifying fibromas of the lower extremity with calcium sulfate grafts in skeletally immature patients. Eur J Orthop Surg Traumatol 28(2):291-297

11. Luthringer BJ, Ali F, Akaichi H et al (2013) Production, characterisation, and cytocompatibility of porous titanium-based particulate scaffolds. J Mater Sci Mater Med 24(10):2337-2358

12. Roddy E, DeBaun MR, Daoud-Gray A et al (2018) Treatment of critical-sized bone defects: clinical and tissue engineering perspectives. Eur J Orthop Surg Traumatol 28(3):351-362

13. Vasita R, Katti DS (2006) Nanofibers and their applications in tissue engineering. Int J Nanomed 1(1):15-30

14. D'Antimo C, Biggi F, Borean A et al (2017) Combining a novel leucocyte platelet-concentrated membrane and an injectable collagen scaffold in a single-step AMIC procedure to treat chondral lesions of the knee: a preliminary retrospective study. Eur J Orthop Surg Traumatol 27(5):673-681 